\title{
High-Sensitivity C Reactive Protein: Associations with Cardiovascular Risk Factors and Tracking in Female Adolescents and Young Adults
}

\author{
John A. Morrison, ${ }^{1}$ Charles J. Glueck, ${ }^{2}$ Stephen R. Daniels, ${ }^{3}$ Ping Wang, ${ }^{2}$ \\ Davis M. Stroop, ${ }^{4}$ and Paul S. Horn ${ }^{5,6}$ \\ ${ }^{1}$ Division of Cardiology, Cincinnati Children's Hospital Medical Center, Cincinnati, OH 45229-3039, USA \\ ${ }^{2}$ Cholesterol Center, Jewish Hospital of Cincinnati-Mercy Health Partners, ABC Building, 3200 Burnet Avenue, \\ Cincinnati, OH 45229, USA \\ ${ }^{3}$ Children's Hospital, Denver, CO 80045, USA \\ ${ }^{4}$ Division of Hematology, Cincinnati Children's Hospital Medical Center, Cincinnati, OH 45229-3039, USA \\ ${ }^{5}$ Department of Mathematical Sciences, University of Cincinnati, Cincinnati, OH 45221, USA \\ ${ }^{6}$ Psychiatry Service, Veterans Affairs Medical Center, Cincinnati, OH 45220, USA
}

Correspondence should be addressed to Charles J. Glueck, cjglueck@health-partners.org

Received 24 November 2010; Accepted 30 December 2010

Academic Editors: C. D. Berkowitz and S. A. Sarker

Copyright (c) 2011 John A. Morrison et al. This is an open access article distributed under the Creative Commons Attribution License, which permits unrestricted use, distribution, and reproduction in any medium, provided the original work is properly cited.

\begin{abstract}
Objective. We assessed adolescent anthropometry, lipids, insulin, glucose, and blood pressures to identify factors associated with high-sensitivity C-reactive protein (hsCRP) and its tracking in young adults. Methods. Ten-year prospective study of 589 schoolgirls, 321 black, 268 white. Results. HsCRP did not differ $(P>.08)$ by race or oral contraceptive use. HsCRP tracked from age 16 to $25(r=0.77), 16$ to $26(r=0.50), 24$ to $26(r=0.66)$, and 25 to $26(r=0.71)$, all $P \leq .02$. By stepwise regression, at age 16, waist circumference accounted for $44.8 \%$ of hsCRP variance; BMI accounted for $33.1 \%, 34.4 \%$, and $31.1 \%$ at ages 24 , 25 , and 26, $P<.0001$ for all. Changes in cholesterol and BMI were associated with change in hsCRP from age 24-26 (partial $R^{2}=12.3 \% P<.0001,6.6 \% P=.0012$ ). Changes in BMI and triglyceride (partial $\left.R^{2}=8.5 \% P=.0001,3.3 \%, P=.0045\right)$ were associated with change in hsCRP from age 25 to 26. Conclusions. HsCRP tracks from age 16 to 26, with BMI, waist circumference, and cholesterol as major determinants.
\end{abstract}

\section{Introduction}

The anatomic and pathophysiological genesis of atherosclerosis is in childhood [1-5]. The traditional Framingham risk factors (hyperlipidemias, blood pressure, obesity, diabetes, and cigarette smoking) have been used as the starting point for evaluating individual risk to vascular disease, and each factor is strongly associated with increased risk to coronary heart disease (CHD) in adults and, in children, associated with family history of CHD [6-9]. It has been estimated that the traditional CHD risk factors may explain about $50 \%$ of CHD morbidity and mortality [9], eliciting efforts to identify additional predictive factors, such as high-sensitivity $\mathrm{C}$ reactive protein (hsCRP) [10].
Biomarkers predictive of inflammatory processes in acute clinical settings (e.g., during infection or after traumas) have become valuable predictors of future vascular events [11]. This primal organism response to invading pathogens, trauma, or chronic inflammation presumably represents a protective mechanism and involves a cascade of thrombotic and inflammatory factors of variable expression [11]. In the chronic state, acute phase reactants have become important predictors of future vascular events: thus, baseline serum high-sensitivity c-reactive protein (hsCRP) values have been correlated with the development of CHD in healthy men $[11,12]$ and women $[11]$ in the Harvard Study population, corroborated by other groups $[13,14]$. In a prospective study of 506 men who had fatal CHD or had a nonfatal 
myocardial infarction compared to 1025 men free of CHD, men in the top third for entry hsCRP had an odds ratio for CHD of 2.13 (95\% confidence interval 1.38-3.28) after age, town, smoking, vascular risk factors, and indicators of socioeconmic status were adjusted for [15]. The cascade of TNF- $\alpha$ and IL- 6 elevation leads to increments in hsCRP and fibrinogen, with resulting adhesion molecule expression, and may facilitate development of type 2 diabetes mellitus (T2DM) as well as CHD [16].

Cross-sectional studies in children have shown a strong association between hsCRP and obesity, triglycerides, HDL cholesterol, diastolic blood pressure, and fibrinogen [1720]. Longitudinal data from the Young Finns Study [21] showed that hsCRP tracked significantly from ages 3-18 years (at entry) to ages 24-39 years. Tracking was greater in the older children and in women than men [21]. By multivariate analysis, adult carotid artery intimal medial thickness (cIMT) was associated with childhood elevated systolic blood pressure, high LDL cholesterol, and smoking, but not with childhood hsCRP [21]. Järvisalo et al. [22], however, showed that elevated hsCRP levels in children are associated with increased cIMT as well as with decreased brachial artery flow-mediated dilatation, and Zieske et al. [23] reported that serum hsCRP is independently associated with advanced atherosclerosis in youth. Thus, in aggregate, these studies suggest that measurement of hsCRP in childhood [21-23] might predict adult cardiovascular risk.

The National Growth and Health Study (NGHS) [24], its ancillary studies, and the extension of the followup of the Cincinnati clinic cohort by investigator-initiated research projects, provide an opportunity to assess hsCRP tracking in black and white females from mid-adolescence to young adulthood and to identify factors associated with high concentrations of hsCRP and with its tracking.

\section{Materials and Methods}

2.1. The Study Population. The NGHS has been described previously [24]. Briefly, NGHS was a 10-year (1987-1997) multicenter cohort study to explicate origins of black-white disparities in obesity and its effects on cardiovascular (CVD) risk factors in women [24]. Race was self-declared and enrollment was restricted to 9- and 10-year old girls from racially concordant households, that is, to girls who said they were black or white and whose parents or guardians said that they were black or white, respectively. In ancillary projects, the Cincinnati clinic measured fasting insulin at Visit 7 (mean \pm SD age $16 \pm 1$ ), in addition to the NGHS variables-lipid profiles, apo A1, systolic and diastolic blood pressure [25]. At age 16, hsCRP was measured in a subset of participants as a pilot project. After completion of NGHS, the Cincinnati clinic extended followup with measurement of insulin to age 25 and collected hsCRP at ages 24, 25, and 26.

In the NGHS [25], and its extension, procedures followed were in accordance with the ethical standards of the Institutional Review Boards of the Centers, who approved the study. Signed informed consent was obtained from the girls' parents or guardians and assent from the girls.
2.2. Laboratory and Clinical Measurements. Methods for measurement of lipids, fasting serum insulin, glucose, height, weight, waist circumference, and systolic and diastolic blood pressure have been previously described [24, 25]. Fasting glucose was measured at ages 10 and 19-24, insulin at ages 10,16 , and 19-25.

At each visit, girls were asked whether they were taking a birth control pill, since hsCRP may be elevated by estrogenprogestin oral contraceptives [26].

The NGHS used BMI to assess overweight annually according to a standard protocol [24], as recommended by several expert panels [27-29] and waist circumference as an indicator of fat patterning.

Using criteria previously described [30], NGHS subjects having fasting blood glucose $\geq 126 \mathrm{mg} / \mathrm{dl}$ [31] at age 10 or type $1 \mathrm{DM}$ at any time from age 10 through age 25 were excluded $(n=7)$ from the analysis sample for this report. Diagnosis of diabetes was based on WHO criteria, fasting glucose $\geq 126 \mathrm{mg} / \mathrm{dl}$, and self-reported diabetes with treatment by a physician [31]. We did not have measurement of C-peptides as well as diabetes autoantibody levels, gold standard methods [32] to distinguish type 1 from type 2 diabetes.

Fasting serum insulin levels (competitive proteinbinding radioimmunoassay) were measured after an overnight fast ( $\geq 8 \mathrm{hr}$ ) using the Michigan Diabetes Research and Training Center (Ann Arbor) at age, and using the Endocrine Lab at the University of Cincinnati/Children's Medical Center at ages 16,19, and 20 to 25.

HsCRP was measured using the $\mathrm{N}$ high-sensitivity latexenhanced immunonephelometric (BN 100 nephelometer, Dade Behring) assay.

2.3. Statistical Methods. Since hsCRP was not normally distributed, black-white differences in hsCRP and differences between birth control pill users and nonusers were assessed by Wilcoxon rank sum tests. For assessment of changes in hsCRP and for regression analyses, hsCRP was transformed in logarithm scale.

Spearman correlations were used to assess the tracking of hsCRP from age 16 to 26 years, Table 1. After adjusting for age, race, BMI, waist circumference, insulin, maximum glucose, total cholesterol, HDL cholesterol, LDL cholesterol, triglyceride, and systolic and diastolic blood pressure, Spearman partial correlations were also calculated to assess the tracking of hsCRP from ages 24 to 26 , and 25 to 26 , Table 1.

Spearman correlations were used to assess the associations of hsCRP with cardiovascular risk factors at ages $16,24,25$, and 26 years including age, race, BMI, waist circumference, insulin, total cholesterol, triglyceride, HDL cholesterol, LDL cholesterol, systolic blood pressure, diastolic blood pressure, and maximum glucose, Table 2. The Hochberg-Benjamini [33] False Discovery Rate (FDR) for multiple comparisons was used, Table 2.

After $\log$ transformation of hsCRP, all of the variables from Table 2 were incorporated as candidate explanatory variables into stepwise regression models with hsCRP as the dependent variable at ages 16, 24, 25, and 26, Table 3. 
TABLE 1: Simple and partial correlations of hsCRP from age 16 to age 26 years.

\begin{tabular}{lcccc}
\hline & At age 16 & At age 24 & At age 25 & At age 26 \\
\hline hsCRP first measured & $n=37$ & $n=166$ & $n=366$ & $n=20$ \\
\hline Spearman correlation & & & $n=22$ & $n=21$ \\
with hSCRP measured & $n=37$ & $n=6$ & $r=0.77$ & $r=0.50$ \\
at age 16 & & $P<.0001$ & $P=.021$ \\
\hline & & & $n=147$ \\
Spearman correlation & $n=172$ & $n=3$ & $r=0.66$ \\
with hSCRP measured & & & partial* \\
at age 24 & & & $r=139$ \\
& & & $P<.0001$ \\
& & & $n=225$ \\
& & & $r=0.71$ \\
& & $n=391$ & partial* \\
Spearman correlation & & & $n=223$ \\
with hSCRP measured & & & $r=0.54$ \\
at age 25 & & & $P<.0001$ \\
\hline & & & $n=392$ \\
\hline hsCRP measured at & & & \\
age 26 & & & \\
\hline
\end{tabular}

* partialed by age, race, BMI, total, HDL, and LDL cholesterol, triglyceride, insulin, systolic blood pressure and diastolic blood pressure, all at the same age, and by maximum glucose measure from age 10 to age 24 years.

Spearman correlations were used to assess relationships of change in hsCRP (in logarithm scale) from ages 24 to 26 and from 25 to 26 with changes in cardiovascular disease variables: age, BMI, total cholesterol, triglyceride, HDL cholesterol, LDL cholesterol, systolic blood pressure, and diastolic blood pressure during the same period, Table 4. The FDR approach [33] for multiple comparisons was again used, Table 4.

All of the variables from Table 4 were incorporated as candidate explanatory variables into stepwise regression models with change in hsCRP (in logarithm scale) from age 24 to 26 and from 25 to 26 as dependent variables, Table 5.

\section{Results}

HsCRP did not differ between birth control pill users versus nonusers, $P \geq .28$ and did not differ by race, $P \geq .09$. Hence, data are presented for the total cohort without subcategorization by birth control pill use or race.

There were no differences $(P>.05)$ in hsCRP or CVD risk factors between girls with $\geq 2$ sequential hsCRP measurements and those with only 1 measurement.

Median and interquartile range for hsCRP at age 16 were $1.5(0.7-5.6)$, at age $24,2.0(0.7-4.0)$, at 25, $1.7(0.6-4.2)$, and at $26,1.7(0.6-4.6) \mathrm{mg} / \mathrm{L}$.

3.1. Tracking of hsCRP from Age 16 to Age 26 Years. HsCRP was measured in 589 girls, 321 black, and 268 white. Subsets of the cohort had hsCRP measured at different ages, 1, 2, 9, and 10 years apart, making possible studies of hsCRP tracking and factors associated with it, Table 1. Thus, there were 37 girls having hsCRP measured at age 16 (18 black, 19 white) with subsequent measurements at age $24(n=$ $6), 25(n=22)$ and $26(n=21)$, Table 1 . In addition, 172 girls (91 black, 81 white) had hsCRP measured at age 24 , with subsequent measures at age $25(n=3)$ and at age $26(n=147)$, Table 1 . Additionally, hsCRP was measured at age 25 in 391 girls (217 black, 174 white) and subsequently at age 26 in 225 of these girls, Table 1. HsCRP was measured at age 26 in 392 girls (215 black, 177 white), Table 1.

Age 16 hsCRP correlated with subsequent measures at ages $25(r=0.77, P<.0001)$ and $26(r=0.50, P=.02)$, Table 1. Age 24 hsCRP correlated with levels at age $26(r=$ $0.66, P<.0001)$ and age 25 hsCRP correlated with levels at age $26(r=0.71, P<.0001)$, Table 1 .

After adjusting for age, race, BMI, insulin, maximum glucose, total cholesterol, triglyceride, HDL cholesterol, LDL cholesterol, and systolic and diastolic blood pressure, hsCRP at age 24 remained highly correlated with levels at age 26 (partial $r=0.63, P<.0001$ ), as did hsCRP at age 25 with levels at age 26 (partial $r=0.54, P<.0001$ ), Table 1 .

Of 43 girls with hsCRP at age 24 in the top $30 \%$ of the distribution, $24(56 \%)$ remained in the top $30 \%$ at age 26 , while 26 of 41 (63\%) with hsCRP at age 24 in the bottom $30 \%$ of the distribution remained in the bottom $30 \%$ at age 26 . Of 62 girls in the hsCRP top 30\% at age 25, $42(68 \%)$ remained in the top $30 \%$ at age 26 , while 49 of $72(68 \%)$ originally in the bottom $30 \%$ at age 25 remained there at age 26 .

3.2. Correlates of hsCRP. Of the 37 girls at age 16, significant positive bivariate correlates with hsCRP included BMI, waist circumference, insulin, and maximum glucose, Table 2.

At ages 24, 25, and 26, significant bivariate correlates with hsCRP included BMI, waist circumference, insulin, total cholesterol, triglyceride, HDL cholesterol, LDL cholesterol, systolic blood pressure, diastolic blood pressure, and maximum glucose, Table 2 . Age was significantly correlated with hsCRP at age 26.

By stepwise regression, waist circumference (age 16, partial $R^{2}=44.8 \%$ ) and BMI (ages 24, 25, and 26, partial $\left.R^{2}=33.1 \%, 34.4 \%, 31.1 \%\right)$ were the major significant $(P<$ .0001 ) explanatory variables for hsCRP, Table 3 . At ages 24 and 25, total cholesterol levels were significant explanatory variables, accounting for $2.5 \%$ and $1.9 \%$ of hsCRP variance, Table 3. At age 26, triglyceride accounted for 3.5\% of hsCRP variance, Table 3 . With the exception of inclusion of race as a significant explanatory variable at age 26 , the results in Table 3 were identical to results if only those variables which had significant bivariate correlations in Table 2 were included as candidate explanatory variables (data not shown).

Race was unrelated to change in hsCRP from age 24 to 26, and from age 25 to 26, Table 4. Changes in BMI, waist circumference, total cholesterol, triglyceride, and LDL cholesterol were positively and significantly related to changes in hsCRP from ages 24 to 26, Table 4 . Changes in BMI, waist circumference, total cholesterol, and triglyceride were positively and significantly related to changes in hsCRP from age 25 to 26, Table 4 . 


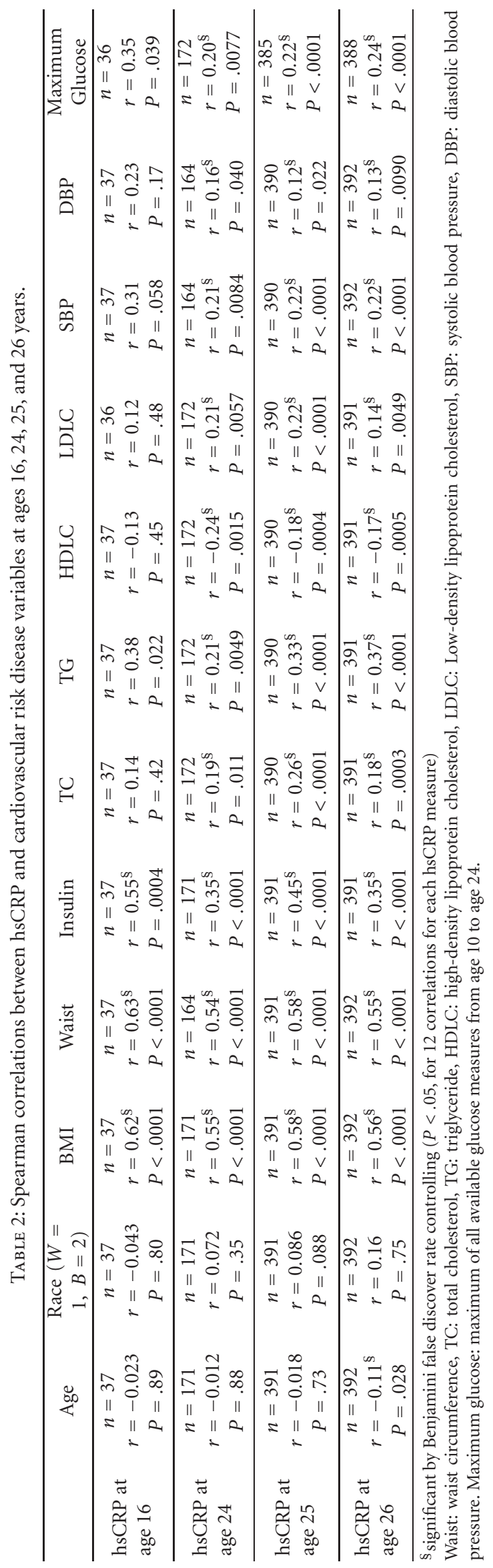


TABLE 3: Stepwise regression of hsCRP at ages 16, 24, 25, and 26 hsCRP are log transformed. Explanatory variables include race, age, BMI, waist circumference, insulin, TC, TG, HDLC, LDLC, systolic, and diastolic blood pressures all at the age when hsCRP measured, and maximum glucose (from age 10 to age 24 ). Significant levels: SLE $=0.15$, SLS $=.05$ in stepwise selection.

\begin{tabular}{|c|c|c|c|c|}
\hline Dependent variable & Significant explanatory variable & $\operatorname{sign}$ & Partial $R^{2}$ & $P$ \\
\hline hsCRP at age 16 (35 observations used) & waist circumference & + & $44.8 \%$ & $<.0001$ \\
\hline \multirow{2}{*}{ hsCRP at age 24 (164 observations used) } & BMI & + & $33.1 \%$ & $<.0001$ \\
\hline & $\mathrm{TC}$ & + & $2.5 \%$ & .014 \\
\hline \multirow{3}{*}{ hsCRP at age 25 (383 observations used) } & BMI & + & $34.4 \%$ & $<.0001$ \\
\hline & $\mathrm{TC}$ & + & $1.9 \%$ & .0008 \\
\hline & waist circumference & + & $0.8 \%$ & .030 \\
\hline \multirow{4}{*}{ hsCRP at age 26 (386 observations used) } & BMI & + & $31.1 \%$ & $<.0001$ \\
\hline & TG & + & $3.5 \%$ & .0002 \\
\hline & Age & - & $2.1 \%$ & .0010 \\
\hline & Race $(W=1, B=2)$ & - & $1.1 \%$ & .0092 \\
\hline
\end{tabular}

TC: total cholesterol, TG: triglyceride, HDLC: HDL cholesterol, LDLC: LDL cholesterol.

After including all of the candidate explanatory variables of Table 4 into a stepwise regression model, change in total cholesterol and change in BMI were significant explanatory variables for change in hsCRP from age 24 to 26 (partial $R^{2}=12.3 \%, 6.6 \%$ ), Table 5. Changes in BMI (partial $R^{2}=$ $8.5 \%$ ) and change in TG (partial $R^{2}=3.3 \%$ ) were significant explanatory variables for change in hsCRP from age 25 to 26 , Table 5. The results in Table 5 were identical to results if only those variables which had significant bivariate correlations in Table 4 were included as explanatory variables (data not shown).

\section{Discussion}

The chronic expression of acute phase proteins, in the stable, ostensibly nonacute setting, is of unclear etiology. Such proteins could be present in excess due to (a) incomplete resolution of a previous acute episode, (b) ongoing monocyte/macrophage activation, and/or (c) enhanced baseline production determined by coordinate genetic control. Activation of the monocyte/macrophage in the acute setting may be among the first dominoes in the inevitable cascade of expressed cytokines and growth factors [34, 35]. Activated monocytes produce TNF and IL-1-like cytokines, which lead to the full acute phase reaction, including hsCRP (17).

In adults, statins lower hsCRP concurrently with LDL cholesterol, and hsCRP levels achieved on statin therapy are predictive of CVD event rates irrespective of the lipid endpoint used [10]. To test the hypothesis whether or not subjects with normal LDL cholesterol but elevated hsCRP represent a population at increased risk that might benefit from statin treatment, the JUPITER trial [10] randomized 17,802 apparently healthy persons with elevated hsCRP to $20 \mathrm{mg}$ Rosuvastatin daily or placebo. The rates of the primary endpoint (composite of nonfatal myocardial infarction, nonfatal stroke, hospitalization for unstable angina, revascularization, and confirmed death from cardiovascular causes) were 0.77 and 1.36 per 100 person-years of followup in the Rosuvastatin and placebo groups, respectively. Relative risk reduction was $44 \%$ [10]. It was postulated that hsCRP more accurately selected high-risk subjects than LDL cholesterol due to hsCRP's association with multiple CVD risk factors, thus representing an aggregate-integrated marker of the total inflammatory burden of an individual [10]. While inflammation is a crucial component of atherothrombosis [36] and patients with elevated hsCRP and low or intermediate LDL cholesterol levels $[10,37]$ are at increased vascular risk, it is not known whether inhibition of inflammation per se will lower vascular event rates [36]. A direct test of the inflammatory hypothesis of atherothrombosis requires an agent that inhibits inflammation without affecting other components of the atherothrombotic process, particularly LDL cholesterol [36]. The forthcoming cardiovascular inflammation reduction trial (CIRT) will randomize 7000 stable coronary artery disease patients with persistent elevations of hsCRP to placebo or very low-dose methotrexate $(10 \mathrm{mg}$ weekly), a proven anti-inflammatory regimen that reduces TNF-alpha, IL-6, and CRP levels and is in wide use among rheumatoid arthritis patients [36]. The CIRT trial [36], if successful, would help confirm the inflammatory hypothesis of atherothrombosis and open innovative, anti-inflammatory approaches to the treatment and prevention of cardiovascular disorders.

It is not yet known whether hsCRP is a risk factor for cardiovascular disease independent of other known risk factors $[10,37]$, nor is it well understood how long hsCRP would need to be normalized independent of changes in other CVD risk factors to reduce CVD events [36]. Moreover, it is not yet known whether hsCRP, a $\$ 43$ test, is useful in assessing childhood antecedents of young adult cardiovascular risk above and beyond conventional childhood CVD risk factors [21], and whether high hsCRP is reversible with childhood therapeutic interventions. Although Karakas and Koenig reported that birth control pill use elevated hsCRP [38], it had no significant effect on hsCRP in our current study. Juonala et al. [21] reported significant 21-year tracking of hsCRP from childhood and adolescence into the fourth decade, but hsCRP was not independently associated with young adulthood carotid intimal-medial thickness (cIMT). Järvisalo et al. [22], however, showed that elevated hsCRP 


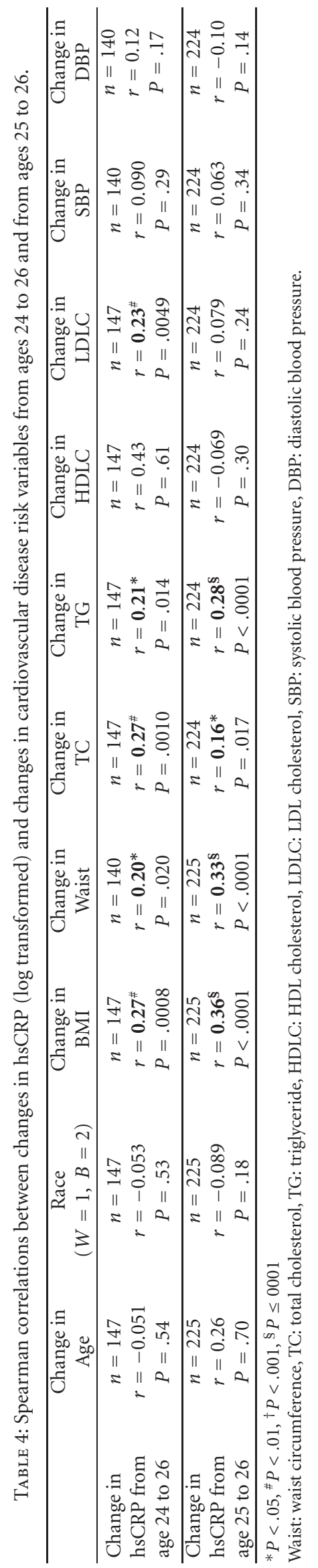


TABLE 5: Change in hsCRP (logarithm transformed) from age 24 to age 26 and from age 25 to 26.

\begin{tabular}{|c|c|c|c|c|}
\hline Dependent variable & Significant explanatory variable & sign & Partial $R^{2}$ & $P$ \\
\hline \multirow{2}{*}{ Change in hsCRP from age 24 to age 26 ( 139 observations used) } & Change in TC & + & $12.3 \%$ & $<.0001$ \\
\hline & Change in BMI & + & $6.6 \%$ & .0012 \\
\hline \multirow{2}{*}{ Change in hsCRP from age 25 to age 26 ( 223 observations used) } & Change in BMI & + & $8.5 \%$ & .0001 \\
\hline & Change in TG & + & $3.3 \%$ & .0045 \\
\hline
\end{tabular}

Dependent variable: hsCRP (logarithm transformed) change.

Explanatory variables include: race, change in age, change in BMI, changes in waist circumference, changes in TC, TG, HDLC, and LDLC, and change in blood pressure during the same period.

Significant levels: SLE $=.15$, SLS $=.05$.

levels in children were associated with increased cIMT and decreased brachial artery flow-mediated dilatation, a marker of the loss of vascular flexibility. In addition, Zieske et al. [23] reported that serum hsCRP is independently associated with advanced atherosclerosis in youth.

In the current study, there was significant tracking of hsCRP across several years from age 24 to $26(r=0.66, P<$ $.0001)$ and from age 25 to $26(r=0.71, P<.0001)$. Similarly strong long-term correlations were found from age 16 to ages $25(r=0.77, P<.0001)$ and $26(r=0.50, P=.02)$. These tracking coefficients remained significant after adjusting for multiple CVD risk factors; hsCRP at age 24 remained highly correlated with levels at age 26 (partial $r=0.63, P<.0001$ ), as did hsCRP at age 25 with levels at age 26 (partial $r=$ $0.54, P<.0001)$. Exemplifying this strong tracking of hsCRP, 24 of 43 girls (56\%) with top 30\% hsCRP at age 24 remained in the top $30 \%$ at age 26 , while 26 of 41 girls (63\%) with bottom $30 \%$ hsCRP at age 24 remained in the bottom $30 \%$ at age 26 . Of 62 girls in the top $30 \%$ of the hsCRP distribution at age $25,42(68 \%)$ remained in the top $30 \%$ at age 26 , while 49 of $72(68 \%)$ originally in the bottom $30 \%$ at age 25 remained there at age 26 .

With exception of the report by Juonala et al. [21], tracking of hsCRP during childhood and into young adulthood has not yet been reported. With 21-year followup from childhood to young adulthood, Juonala et al. [21] reported significant tracking between childhood and adult hsCRP levels, highest in 18 years olds at study entry, $r=0.47$ in females, 0.32 in males, and $P<.0001$ for both. For the total cohort, the 21-year tracking correlation for hsCRP was 0.29 [21]. Congruent with the findings of the current study, the association between childhood and adult hsCRP levels in Finns [21] was independent of serum lipids, blood pressure, smoking, obesity indices, and insulin.

Significant tracking of hsCRP from childhood into young adulthood speculatively raises the issue whether childhood hsCRP can predict adult atherosclerosis or CVD events independent of traditional cardiovascular risk factors (HDL and LDL cholesterol, triglyceride, blood pressure, and obesity). In the study by Juonala et al. [21], however, childhood hsCRP was not independently associated with young adulthood carotid IMT. In 8091, subjects with hsCRP $\geq 2 \mathrm{mg} / \mathrm{L}$ randomized to placebo in the JUPITER (Rosuvastatin) trial, over a 4-year period, the intraclass correlation for repeated hsCRP measurements was 0.54 (95\% CI 0.53-0.55) without adjustment and 0.50 (95\% CI 0.49-0.51) after adjustment for demographic, lifestyle, and comorbidity determinants [39], Glynn et al. [39] concluded that "... concentrations of hsCRP show strong tracking, even after selection of individuals with initially high values. Without statin therapy, increased concentrations of hsCRP generally remain high over time."

Congruent with the findings of Soriano-Guillén et al. [20], in the current study, at age 16, hsCRP was significantly positively correlated with BMI, waist circumference, and insulin. By stepwise regression, waist circumference and BMI were the major significant $(P<.0001)$ explanatory variables for hsCRP, with waist circumference accounting for $44.8 \%$ of the variance of hsCRP at age 16, and BMI accounting for $33.1 \%$ of variance of hsCRP at age $24,34.4 \%$ at age 25 , and $31.1 \%$ at age 26 . The consistent, independent, and significant association of BMI with hsCRP from age 16 to 26 in our current study emphasizes the pediatric and young adult cardiovascular ramifications of obesity [1]. In a recently reported 23.9 year followup study from age 11.3 years of 4857 American Indian children without diabetes, endogenous death rates among children with the highest quartile of BMI were more than double those among children in the lowest BMI quartile (incidence-rate ratio, 2.30, 95\% confidence interval 1.46 to 3.62 ) [1].

Our report is limited by the small number of girls $(n=$ 37 ) that had hsCRP measurements at age 16 and then again at ages 25 and 26, thus limiting the information on tracking from adolescence into young adulthood. However, tracking data from age 24 to 26 was available in 147 girls and in 225 from age 25 to 26 .

In adults, hsCRP levels have been correlated with the development of CHD in healthy men [12] and women $[11,13,14]$. In adults, hsCRP levels are associated with the traditional CHD risk factors, but also confer independent information about CHD status and future risk [10, 40]. High childhood hsCRP might be viewed as a useful index of childhood obesity that would affect progression to future premature death from endogenous causes [1] and to atherosclerotic disease $[18,41]$.

\section{Acknowledgments}

This research was supported in part by NIH- HL55025, 48941, HL52911 and HL66430 [Drs. Morrison and Daniels], and by the Lipoprotein Research Fund of the Jewish Hospital 
of Cincinnati [Dr. Glueck], by grant $9750129 \mathrm{~N}$ from the American Heart Association [Dr. Morrison], by the Taft Research Fund [Dr. Horn].

\section{References}

[1] G. S. Berenson and S. R. Srinivasan, "Emergence of obesity and cardiovascular risk for coronary artery disease: the Bogalusa Heart Study," Preventive Cardiology, vol. 4, no. 3, pp. 116-121, 2001.

[2] M. D. Haust, "The genesis of atherosclerosis in pediatric agegroup," Pediatric Pathology, vol. 10, no. 1-2, pp. 253-271, 1990.

[3] H. C. McGill Jr., C. A. McMahan, E. E. Herderick, G. T. Malcom, R. E. Tracy, and J. P. Strong, "Origin of atherosclerosis in childhood and adolescence," American Journal of Clinical Nutrition, vol. 72, no. 5, pp. 1307S-1315S, 2000.

[4] C. A. McMahan, S. S. Gidding, Z. A. Fayad et al., "Risk scores predict atherosclerotic lesions in young people," Archives of Internal Medicine, vol. 165, no. 8, pp. 883-890, 2005.

[5] C. A. McMahan, S. S. Gidding, G. T. Malcom, R. E. Tracy, J. P. Strong, and H. C. McGill Jr., "Pathobiological determinants of atherosclerosis in youth risk scores are associated with early and advanced atherosclerosis," Pediatrics, vol. 118, no. 4, pp. 1447-1455, 2006.

[6] L. H. Kuller, "Cardiovascular disease is preventable among women," Expert Review of Cardiovascular Therapy, vol. 8, no. 2, pp. 175-187, 2010.

[7] J. Stamler, J. D. Neaton, and D. N. Wentworth, "Blood pressure (systolic and diastolic) and risk of fatal coronary heart disease," Hypertension, vol. 13, no. 5, pp. 2-12, 1989.

[8] S. Lamon-Fava, P. W. F. Wilson, and E. J. Schaefer, "Impact of body mass index on coronary heart disease risk factors in men and women: the Framingham Offspring Study," Arteriosclerosis, Thrombosis, and Vascular Biology, vol. 16, no. 12, pp. 1509-1515, 1996.

[9] M. Wei, B. D. Mitchell, S. M. Haffner, and M. P. Stern, "Effects of cigarette smoking, diabetes, high cholesterol, and hypertension on all-cause mortality and cardiovascular disease mortality in Mexican Americans: the San Antonio Heart Study," American Journal of Epidemiology, vol. 144, no. 11, pp. 1058-1065, 1996.

[10] P. M. Ridker, E. Danielson, F. A. Fonseca et al., "Reduction in C-reactive protein and LDL cholesterol and cardiovascular event rates after initiation of rosuvastatin: a prospective study of the JUPITER trial," The Lancet, vol. 373, no. 9670, pp. 11751182, 2009.

[11] P. M. Ridker, C. H. Hennekens, J. E. Buring, and N. Rifai, "C-reactive protein and other markers of inflammation in the prediction of cardiovascular disease in women," New England Journal of Medicine, vol. 342, no. 12, pp. 836-843, 2000.

[12] P. M. Ridker, M. Cushman, M. J. Stampfer, R. P. Tracy, and C. H. Hennekens, "Inflammation, aspirin, and the risk of cardiovascular disease in apparently healthy men," New England Journal of Medicine, vol. 336, no. 14, pp. 973-979, 1997.

[13] L. H. Kuller, R. P. Tracy, J. Shaten, and E. N. Meilahn, "Relation of C-reactive protein and coronary heart disease in the MRFIT nested case-control study," American Journal of Epidemiology, vol. 144, no. 6, pp. 537-547, 1996.

[14] W. Koenig, M. Sund, M. Fröhlich et al., "C-reactive protein, a sensitive marker of inflammation, predicts future risk of coronary heart disease in initially healthy middle-aged men:
Results from the MONICA (monitoring trends and determinants in cardiovascular disease) Augsburg cohort study, 1984 to 1992," Circulation, vol. 99, no. 2, pp. 237-242, 1999.

[15] J. Danesh, P. Whincup, M. Walker et al., "Low grade inflammation and coronary heart disease: prospective study and updated meta-analyses," British Medical Journal, vol. 321, no. 7255, pp. 199-204, 2000.

[16] J. Spranger, A. Kroke, M. Möhlig et al., "Inflammatory cytokines and the risk to develop type 2 diabetes: results of the prospective population-based European Prospective Investigation into Cancer and Nutrition (EPIC)-Potsdam study," Diabetes, vol. 52, no. 3, pp. 812-817, 2003.

[17] N. Mauras, C. DelGiorno, C. Kollman et al., "Obesity without established comorbidities of the metabolic syndrome is associated with a proinflammatory and prothrombotic state, even before the onset of puberty in children," Journal of Clinical Endocrinology and Metabolism, vol. 95, no. 3, pp. 1060-1068, 2010.

[18] S. P. Yang, C. X. Gong, B. Y. Cao, and C. Yan, "Relationship between serum high-sensitivity C-reactive protein and obesity and impaired glycose metabolism in children and adolescents," Zhonghua Er Ke Za Zhi, vol. 44, no. 12, pp. 933-936, 2006.

[19] O. Guran, F. Akalin, C. Ayabakan, F. Y. Dereli, and G. Haklar, "High-sensitivity C-reactive protein in children at risk for coronary artery disease," Acta Paediatrica, International Journal of Paediatrics, vol. 96, no. 8, pp. 1214-1219, 2007.

[20] L. Soriano-Guillén, B. Hernández-García, J. Pita, N. Domínguez-Garrido, G. Del Río-Camacho, and A. Rovira, "High-sensitivity C-reactive protein is a good marker of cardiovascular risk in obese children and adolescents," European Journal of Endocrinology, vol. 159, no. 1, pp. R1-R4, 2008.

[21] M. Juonala, J. S. A. Viikari, T. Rönnemaa, L. Taittonen, J. Marniemi, and O. T. Raitakari, "Childhood C-reactive protein in predicting CRP and carotid intima-media thickness in adulthood: the Cardiovascular Risk in Young Finns Study," Arteriosclerosis, Thrombosis, and Vascular Biology, vol. 26, no. 8, pp. 1883-1888, 2006.

[22] M. J. Järvisalo, A. Harmoinen, M. Hakanen et al., "Elevated serum C-reactive protein levels and early arterial changes in healthy children," Arteriosclerosis, Thrombosis, and Vascular Biology, vol. 22, no. 8, pp. 1323-1328, 2002.

[23] A. W. Zieske, R. P. Tracy, C. A. McMahan et al., "Elevated serum C-reactive protein levels and advanced atherosclerosis in youth," Arteriosclerosis, Thrombosis, and Vascular Biology, vol. 25, no. 6, pp. 1237-1243, 2005.

[24] J. A. Morrison, "Obesity and cardiovascular disease risk factors in Black and White girls: the NHLBI Growth and Health Study," American Journal of Public Health, vol. 82, no. 12, pp. 1613-1620, 1992.

[25] J. A. Morrison, C. J. Glueck, P. S. Horn, G. B. Schreiber, and P. Wang, "Pre-teen insulin resistance predicts weight gain, impaired fasting glucose, and type 2 diabetes at age 18-19 y: a 10-y prospective study of black and white girls," American Journal of Clinical Nutrition, vol. 88, no. 3, pp. 778-788, 2008.

[26] E. A. Lawson, K. K. Miller, V. A. Mathur et al., "Hormonal and nutritional effects on cardiovascular risk markers in young women," Journal of Clinical Endocrinology and Metabolism, vol. 92, no. 8, pp. 3089-3094, 2007.

[27] S. E. Barlow and W. H. Dietz, "Obesity evaluation and treatment: expert Committee recommendations. The Maternal and Child Health Bureau, Health Resources and Services 
Administration and the Department of Health and Human Services," Pediatrics, vol. 102, no. 3, p. E29, 1998.

[28] T. Cole, "Weight-stature indices to measure underweight, overweight, and obesity," in Anthropometric Assessment of Nutritional Status, J. H. Hines, Ed., pp. 83-111, Wiley-Liss, New York, NY, USA, 1991.

[29] R. J. Kuczmarski, C. L. Ogden, S. S. Guo et al., "2000 CDC Growth Charts for the United States: methods and development," Vital and Health Statistics. Series 11, no. 246, pp. 1-190, 2002.

[30] V. De Leo, M. C. Musacchio, G. Morgante, P. Piomboni, and F. Petraglia, "Metformin treatment is effective in obese teenage girls with PCOS," Human Reproduction, vol. 21, no. 9, pp. 2252-2256, 2006.

[31] K. G. M. M. Alberti and P. Z. Zimmet, "Definition, diagnosis and classification of diabetes mellitus and its complications. Part 1: diagnosis and classification of diabetes mellitus. Provisional report of a WHO consultation," Diabetic Medicine, vol. 15, no. 7, pp. 539-553, 1998.

[32] D. Dabelea, R. A. Bell, R. B. D’Agostino Jr. et al., "Incidence of diabetes in youth in the United States," Journal of the American Medical Association, vol. 297, no. 24, pp. 2716-2724, 2007.

[33] Y. Hochberg and Y. Benjamini, "More powerful procedures for multiple significance testing," Statistics in Medicine, vol. 9, no. 7, pp. 811-818, 1990.

[34] H. Baumann and J. Gauldie, "The acute phase response," Immunology Today, vol. 15, no. 2, pp. 74-80, 1994.

[35] P. K. Kim and C. S. Deutschman, "Inflammatory responses and mediators," Surgical Clinics of North America, vol. 80, no. 3, pp. 885-894, 2000.

[36] P. M. Ridker, "Testing the inflammatory hypothesis of atherothrombosis: scientific rationale for the cardiovascular inflammation reduction trial (CIRT)," Journal of Thrombosis and Haemostasis, vol. 7, no. 1, pp. 332-339, 2009.

[37] P. M. Ridker, "Statin therapy for low-LDL, high-hsCRP patients: from JUPITER to CORONA," Clinical Chemistry, vol. 56, no. 4, pp. 505-507, 2010.

[38] M. Karakas and W. Koenig, "CRP in cardiovascular disease," Herz, vol. 34, no. 8, pp. 607-613, 2009.

[39] R. J. Glynn, J. G. MacFadyen, and P. M. Ridker, "Tracking of high-sensitivity C-reactive protein after an initially elevated concentration: the JUPITER study," Clinical Chemistry, vol. 55, no. 2, pp. 305-312, 2009.

[40] H. D. Sesso, J. E. Buring, N. Rifai, G. J. Blake, J. M. Gaziano, and P. M. Ridker, "C-reactive protein and the risk of developing hypertension," Journal of the American Medical Association, vol. 290, no. 22, pp. 2945-2951, 2003.

[41] M. Hiura, T. Kikuchi, K. Nagasaki, and M. Uchiyama, "Elevation of serum C-reactive protein levels is associated with obesity in boys," Hypertension Research, vol. 26, no. 7, pp. 541546, 2003. 


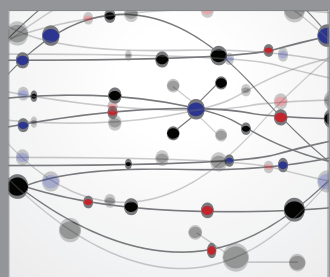

The Scientific World Journal
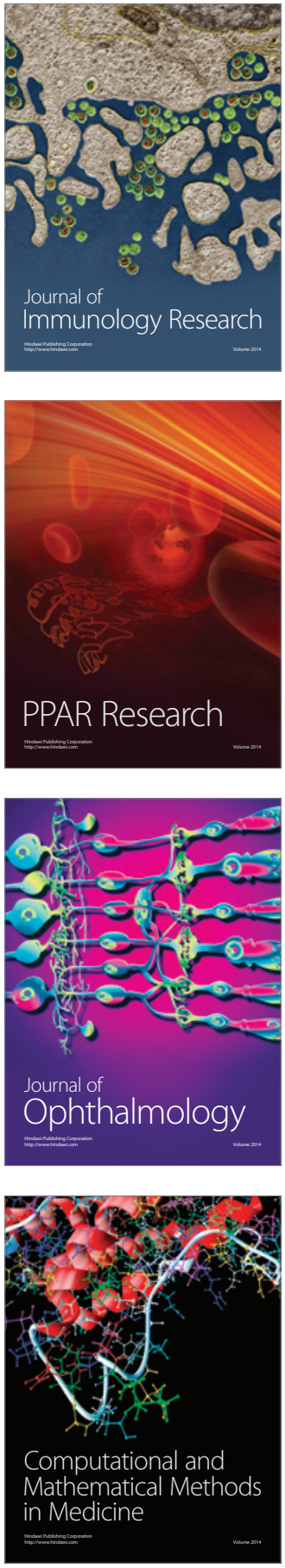

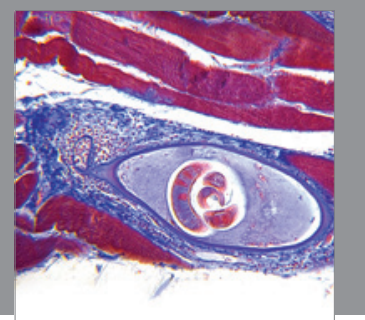

Gastroenterology

Research and Practice
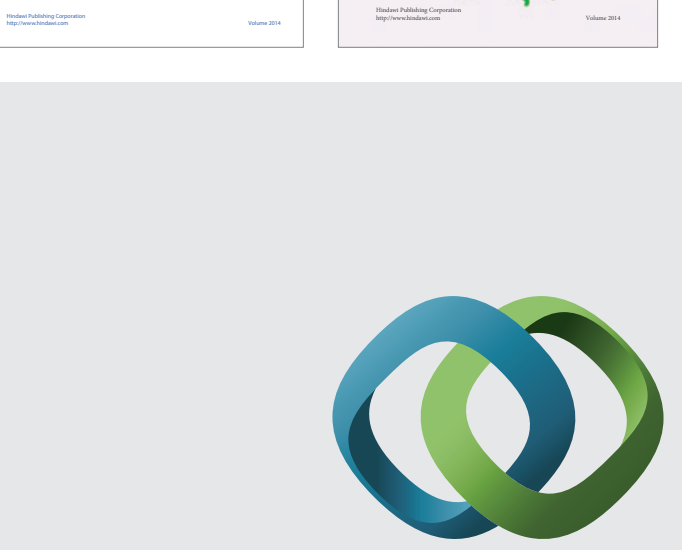

\section{Hindawi}

Submit your manuscripts at

http://www.hindawi.com
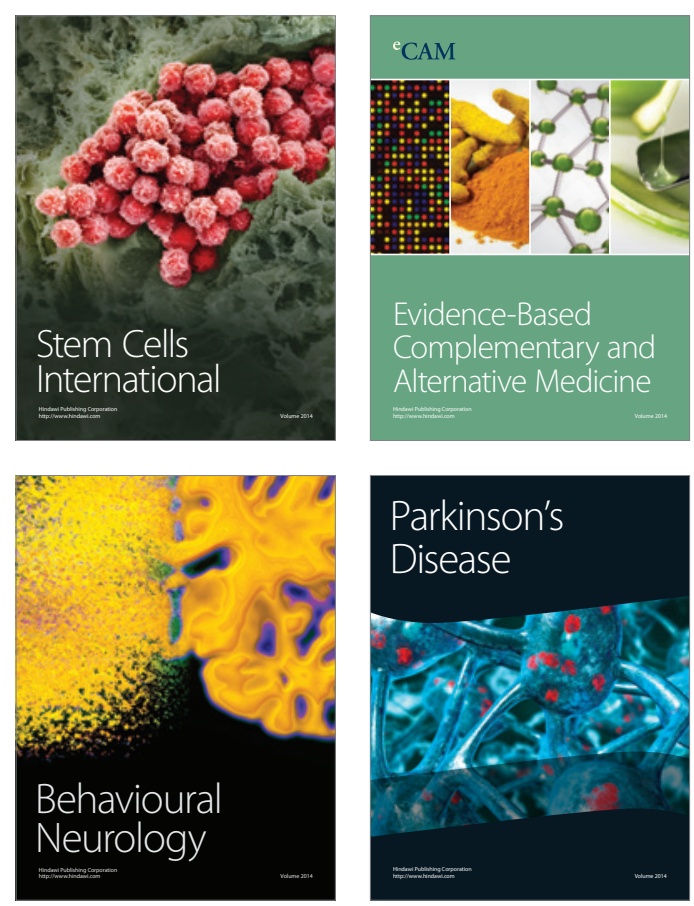

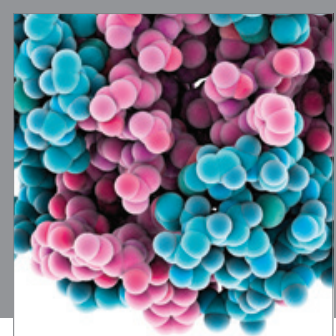

Journal of
Diabetes Research

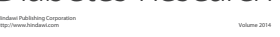

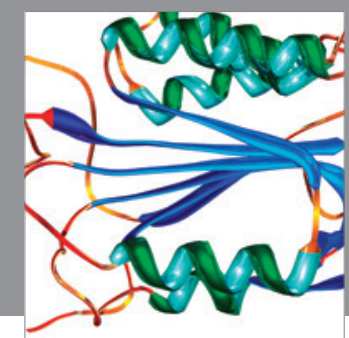

Disease Markers
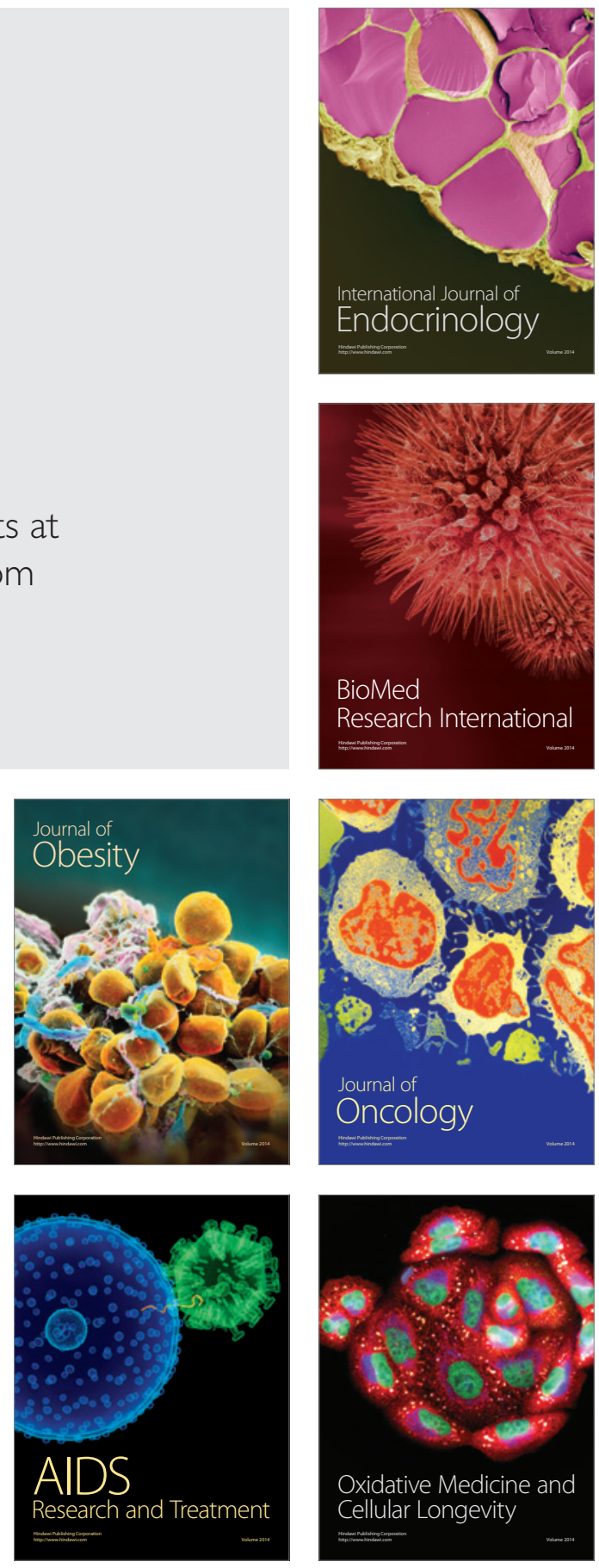